\title{
Electronic Fine Structure in the Electron-Hole Plasma in $\mathrm{SrB}_{6}$
}

\author{
C. O. Rodriguez, ${ }^{1}$ Ruben Weht ${ }^{2}$ and W. E. Pickett ${ }^{3}$ \\ ${ }^{1}$ Instituto de Física de Líquidos y Sistemas Biológicos, Grupo Física del Sólido, Casilla de Correo 565, La Plata 1900, \\ Argentina \\ ${ }^{2}$ Departamento de Física, CNEA, Avda. General Paz 1499 y Constituyentes, 1650 San Martin, Argentina \\ ${ }^{3}$ Department of Physics, University of California, Davis CA 95616
}

\begin{abstract}
Electron-hole mixing-induced fine structure in alkaline earth hexaborides leads to lower energy (temperature) scales, and thus stronger tendency toward an excitonic instability, than in their doped counterparts (viz. $\mathrm{Ca}_{1-x} \mathrm{La}_{x} \mathrm{~B}_{6}, x \approx 0.005$ ), which are high Curie temperature, small moment ferromagnets. Comparison of Fermi surfaces and spectral distributions with de Haas - van Alphen ( $\mathrm{dHvA}$ ), optical, transport, and tunneling data indicates that $\mathrm{SrB}_{6}$ remains a fermionic semimetal down to (at least) $5 \mathrm{~K}$, rather than forming an excitonic condensate. For the doped system the Curie temperature is higher than the degeneracy temperature.
\end{abstract}

The observation of very low moment magnetism (0.07 $\mu_{B}$ per carrier) in the very low carrier density doped semimetal $\mathrm{Ca}_{0.995} \mathrm{La}_{0.005} \mathrm{~B}_{6}$ (one carrier per $6 \times 6 \times 6$ unit cells) at remarkably high temperature $\left(\mathrm{T}_{c}=600 \mathrm{~K}\right)$ by Young et al. [1] has fueled rapid investigation into possible mechanisms for this novel occurrence. [2 14 The focus has been on some sort of excitonic instability, possibly an excitonic condensate that breaks time reversal symmetry and thereby allows ferromagnetism. What has not yet drawn the same attention is the undoped system, which is really the more suitable candidate for an excitonic instability than the doped system. The $\mathrm{DB}_{6}$ hexaborides $(\mathrm{D}=\mathrm{Ca}, \mathrm{Sr}, \mathrm{Ba})$ would be semiconductors with a band gap of $2-3 \mathrm{eV}$ between bonding and antibonding $\mathrm{B}_{6}^{2-}$ $2 p$ bands except for a $\mathrm{D}$ atom $d$ band that dives through the gap from above, with a minimum at the $\mathrm{X}$ point. The result is a very small band overlap $\sim 90 \mathrm{meV}$ at the three Brillouin zone (BZ) edge points X.

The study of instabilities in such semimetals goes back to Keldysh and Kopaev [5] and des Cloizeaux. [6] In the presence of a residual screened Coulomb interaction between the carriers and sufficient nesting, there are possibilities of charge density wave (CDW) or spin density wave (SDW) ('singlet' or 'triplet') instabilities depending on the degree of nesting. Zhitomirsky et al. [2] have suggested that magnetism can appear as a result of consecutive CDW and SDW instabilities in a doped excitonic insulator, with polarization becoming allowed because both inversion and time-reversal symmetries are broken. Barzykin and Gor'kov [3] contend that this model of nested semimetals is instead unstable to the appearance of a superstructure. Balents and Varma [ered the relative stability of the various order parameters involving charge, spin, and X-point pockets and the dependence on doping concentration $x$, and conclude that intrapocket condensation is favored over interpocket pairing.
Each of these discussions presumes the instability of the undoped system, a question that has been revisited by Zhitomirsky and Rice. [7] They emphasize the importance of interpocket scattering processes and reemphasize that the undoped hexaborides should be good candidates for a condensed excitonic state. What we show in this paper, by comparison with band structure derived quantities with the considerable data available [8] for $\mathrm{SrB}_{6}$, is evidence that $\mathrm{SrB}_{6}$ remains a fermionic semimetal as described by conventional band theory down to at least $5 \mathrm{~K}$, and perhaps down to $0.5 \mathrm{~K}$ where a transition to a more conductive (not insulating) phase has been observed. The electronic structure of the $x=0.005$ system is in fact much simpler (in the absence of an excitonic instability) and the doping level and observed Fermi surface volume [9] is consistent with a fully polarized ferromagnet $(\mathrm{FM})$.

The excitonic instability is entirely dependent on the band structure. Some of the band characteristics have been presented by Hasegawa and Yanase [10] and by Massidda et al. [11 and crystal stability has been studied by Ripplinger et al. [12] Here we address the band structure in more detail than heretofore, and find that the calculated energy scales are in excellent agreement with the variety of data 8 on $\mathrm{SrB}_{6}$. The fine structure that we discuss applies to the undoped systems and affects the tendency toward instability, and it can be tested with dHvA data if the system remains fermionic at low temperature $(\mathrm{T})$. We have calculated the band structures of these compounds using the accurate, full potential augmented plane wave method. 13] In these hexaborides the differences between the local density approximation 14 and the generalized gradient approximation 15] (GGA) are small even on the scale of the fine structure we will be discussing, and we use the GGA results. We discuss primarily $\mathrm{SrB}_{6}$ because of the more extensive experimental data, [8] and because when doped, $\mathrm{SrB}_{6}$ also shows 
(as does $\mathrm{BaB}_{6}$ ) a FM phase similar to doped $\mathrm{CaB}_{6}$, but at even higher temperature. [16] We use the measured lattice constant of $4.20 \AA$ and internal parameter (B position along the cubic axis) of 0.203 , the latter confirmed by our total energy minimization. We have checked that spin-orbit coupling has no noticable effect on the results we discuss.

The two bands that overlap at the $\mathrm{X}=(100) \pi / a$ point, shown in Fig. 1, are characterized at the most basic level by longitudinal $\left(m_{l}\right)$ and transverse $\left(m_{t}\right)$ masses, given by $m_{l}=0.50,-2.13$ and $m_{t}=0.21,-0.20$ relative to the free electron mass (hole masses are negative). Thermal masses $\left(m_{t}^{2} m_{l}\right)^{1 / 3}$ are 0.28 and -0.44 respectively. The band overlap ('negative gap') is $90 \mathrm{meV}$ in $\mathrm{SrB}_{6}$. Crossing of the electron $(e)$ and hole $(h)$ bands only occurs precisely along the $\Delta=(\xi, 0,0) \frac{\pi}{a}$ and $\mathrm{Z}=(1, \xi, 0) \frac{\pi}{a}$ lines; elsewhere coupling results in anticrossing. This mixing results in the two overlapping and intersecting ellipsoids (in the absence of coupling) becoming two distinct surfaces as shown in Fig. 2: a circular lens centered on $\Delta$ containing holes, and a ring ("napkin ring") centered at $\mathrm{X}$ containing electrons. Each surface is made of pieces of each of the two ellipsoids, and each contains an $e$ and a $h$ part.

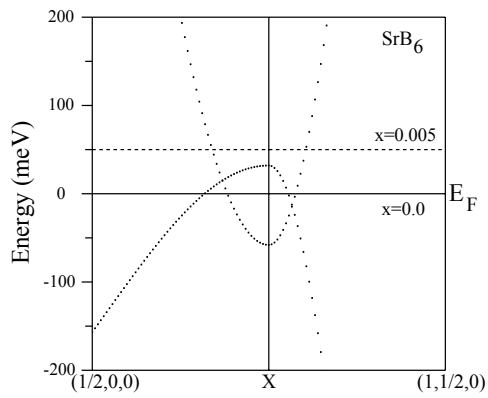

FIG. 1. Calculated band structure near the $\mathrm{X}$ point. The band crossings occur only along these two lines, elsewhere there are anticrossings (not shown). The Fermi levels are shown as horizontal lines for $x=0$ (solid) and $x=0.005$ (dashed). The units of the axes are $\pi / a$.

We have used the condition that the number of electrons must equal the number of holes to determine the intrinsic Fermi energy accurately. The procedure becomes quite delicate, requiring energy grids of spacing $\frac{1}{150} \frac{\pi}{a}$ to represent the hybridization sufficiently accurately. The result is that, whereas the ellipsoids at equal volumes would contain $n_{o}=2 \times 10^{-3}$ carriers, the six lenses and three rings contain only $n_{e} / 2=n_{h} / 2=2.7 \times 10^{-4}$ of the $\mathrm{BZ}$ volume.

The effect of band mixing is most easily seen in the drastic effect it has on the density of states (DOS). As pictured in Fig. 3, instead of simple overlapping $\left(E-E_{o}\right)^{1 / 2}$ edges, there are rather strong peaks near the points of mixing (due to flattened anticrossing bands) with a deep minimum almost at the $x=0$ Fermi level.
This structure makes the DOS at the Fermi level, $\mathrm{N}\left(\mathrm{E}_{F}\right)$, depend much more strongly on carrier concentration than might have been guessed.

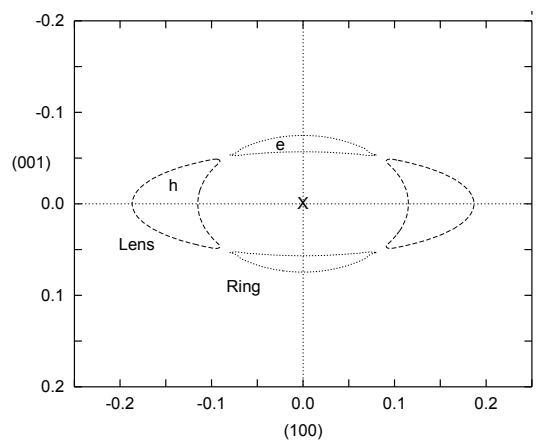

FIG. 2. Cross section of the two Fermi surfaces of undoped $\mathrm{SrB}_{6}$ centered on the $\mathrm{X}$ point. The units of the axes are $\pi / a$. The surfaces enclose electrons $(e)$ and holes $(h)$ surfaces as noted.

In free space this plasma $\left(n_{e}=n_{h}\right)$ would be characterized by an electron gas parameter $r_{s}=60$, which would be an extremely low density plasma. In a solid the effective mass (on average $\left|m^{*}\right| \sim 0.35$, see above) and the background dielectric constant $\epsilon$ rescale the kinetic and potential energies, respectively, and alter the effective density. The value of $\epsilon$ has not been reported. Judging from the fact that $\mathrm{SrB}_{6}$ would have an average direct gap of around $3 \mathrm{eV}$ except for the single conduction band that dips down at $\mathrm{X}$, and that this gap is similar to that of Si $(\epsilon=12)$ but the crystal is less covalent and partially ionic, we estimate $\epsilon \sim 8$ for the background dielectric constant. This value leads to plasma parameters

$$
\begin{aligned}
a_{B}^{*} & =a_{B} \epsilon \frac{m}{m^{*}} \approx 24 a_{B} \approx 13 \AA \\
r_{s}^{*} & =r_{s} \frac{a_{B}}{a_{B}^{*}} \approx 2.5 \\
E_{R}^{*} & =E_{R} \frac{m^{*}}{m} \frac{1}{\epsilon^{2}} \approx 25 \mathrm{meV},
\end{aligned}
$$

here $a_{B}$ is the Bohr radius. The Rydberg $\mathrm{E}_{R}^{*}$, is the exciton binding energy that sets the scale of bandgaps for which a low temperature excitonic instability is possible. Using these values, the $e$ and $h$ densities of $\mathrm{SrB}_{6}$ falls within the range of effective density of alkali metals, but with an excitonic energy scale of $\mathrm{E}_{R}^{*} \sim 25 \mathrm{meV}$ that is two orders of magnitude smaller than in alkali metals. The carrier plasma energy is changed little by this renormalization $]\left(\epsilon^{-1} \mathrm{~m} / \mathrm{m}^{*}\right)^{1 / 2} \sim 1 / 2$ ] and remains on the scale of $100 \mathrm{meV}$. The corresponding Fermi energies that determine the degeneracy temperature are $20 \mathrm{meV}$ for the holes and $15 \mathrm{meV}$ for the electrons, making the degeneracy temperature $\mathrm{T}_{F} \approx 250 \mathrm{~K}$. 


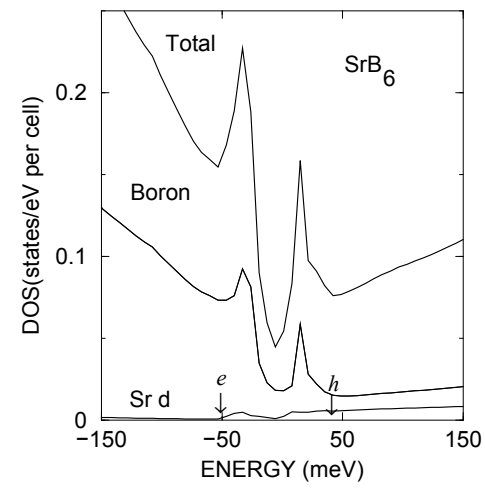

FIG. 3. Density of states of $\mathrm{SrB}_{6}$ near the Fermi level including decomposition into B and Sr contributions. The band anticrossings lead to pronounced structure rather than a simple overlapping to two square-root-like band edges. The $e$ and $h$ band edges are marked.

Barring an excitonic instability, at low temperature $\mathrm{T}<<\mathrm{T}_{F}$ dHvA oscillations will measure the cross sectional areas and cyclotron masses of the extremal Fermi surface orbits perpendicular to the applied field. For the field along a cubic axis, these Fermi surfaces lead to five extremal orbits whose band masses we have calculated from the energy derivative of the cross sectional area $m^{*}=\frac{\hbar^{2}}{2 \pi} d A / d E$. The lens and ring cross sections $O_{\text {lens }, 0}$ and $O_{\text {ring, } 0}$ lie in the plane of Fig. 2. There are three extremal orbits perpendicular to these (circling the $\Delta$ line): $O_{\text {lens }, 1}, O_{\text {ring,in }}$, and $O_{\text {ring,out }}$, where in and out denote the inner and outer ring orbits.

The areas and cyclotron band masses are given in Table I. The values of the three observed frequencies [9] are 30, 48, and 308 Tesla. The first two are in the range of our values, and in fact agree well with the two lens orbits. These two orbits, both areas and masses, arise from the mixing that eliminates the intersections of ellipsoids and creates new orbits that can be seen in Fig. 2. The 308 Tesla orbit is rather large to be accounted for in terms of band structure results; even with magnetic breakdown, the largest orbit (for the cigar ellipsoid) is only 196 Tesla. It is interesting that the available data is consistent with "missing" rings, but it is also possible that ring orbits are simply more difficult to observe.

The distinctive characters of the carriers may play an important role in the behavior of the hexaborides, e.g. by affecting matrix elements or, due to La doping on the alkaline earth sublattice, reducing the mean free path for the electrons much more than for the holes. The band that dips down through the gap has Sr $4 d$ character (Fig. 3 ), and this band forms the electron ellipsoids. Thus the $e$ carriers are a combination of $\mathrm{Sr} d$ and $\mathrm{B} p$, while the $h$ carriers are purely bonding $\mathrm{B} p$ character. Due to the mixing that rehybridizes the bands and leads to the lenses and rings, each Fermi surface is roughly half $e$-like and half $h$-like, and thus partly $\mathrm{Sr} d$ as well as B $p$.

We now compare our results with available data on
$\mathrm{SrB}_{6} \cdot$ [8] The band structures indicate a degeneracy scale $\mathrm{E}^{*}$ of $15-20 \mathrm{meV}$ (degeneracy temperature $\mathrm{T}^{*} \sim 250 \mathrm{~K}$ ), reduced from $600 \mathrm{~K}$ by anticrossing electron and hole bands. Interband transitions in the infrared should peak at the separation of DOS peaks in Fig. 3, near $\omega^{*} \sim$ $40 \mathrm{meV} / \hbar \sim 320 \mathrm{~cm}^{-1}$. The experimental data indicate: (1) a change from metallic slope of resistivity $\rho$ to non-metallic slope at $250 \mathrm{~K} \sim \mathrm{T}^{*}$, consistent with some change as the system evolves from non-degenerate to degenerate (the transport is not understood), (2) a rise in the optical conductivity (at low T) below $300 \mathrm{~cm}^{-1} \sim \omega^{*}$, but the peaking at $40-80 \mathrm{~cm}^{-1}$ (i.e. below $10 \mathrm{meV}$ ) may reflect $\mathrm{k}$-conserving processes not identifiable in the DOS of Fig. 3, (3) a broadening and weakening of this absorption peak on the scale of $200-300 \mathrm{~K} \sim \mathrm{T}^{*},(4)$ a strong onset of interband transitions at $\sim 2 \mathrm{eV}$, roughly consistent with the band structure, (5) negligible electronic contribution to the specific heat above $5 \mathrm{~K}$; the band structure value would not be detectable, (6) tunneling spectra show a conductance minimum at a negative bias of $-40 \mathrm{meV}$ $\approx \hbar \omega^{*}$, and strong $\mathrm{T}$ dependence of the spectrum at -40 $\mathrm{meV}$ and at zero bias. [17] All of these observations are consistent, almost quantitatively, with the band overlap and mixing that we calculate.

There are additional observations [8]: $\rho(T)$ shows structure at $50-60 \mathrm{~K}$, and drops very rapidly by $30 \%$ below $0.5 \mathrm{~K}$, this latter temperature corresponding to an energy scale of $40 \mu \mathrm{eV}$ for which there is no immediate explanation. There is excess specific heat (above the lattice contribution) below $5 \mathrm{~K}$, but with only a small integrated entropy of less than $0.05 \%$ of $R \ln 2$. Except for this low temperature regime, observations seem consistent with a fermionic plasma with band structure as described above.

Now we turn to the doped magnetic system. Doping of $x=0.005$ increases the Fermi level by $50 \mathrm{meV}$ as shown in Fig. 1, for which there is a single ellipsoidal Fermi surface (at each of the $\mathrm{X}$ points) with eccentricity $\eta=\mathrm{k}_{\|} / \mathrm{k}_{\perp}$ $=1.6$. The hole surface vanishes near $x=0.004$. For doped $(x=0.005) \mathrm{CaB}_{6}$, the reported $\mathrm{dHvA}$ frequencies of $350 \mathrm{~T}$ and $495 \mathrm{~T}$ [9] are consistent with such ellipsoids with eccentricity $\eta=495 / 350=1.4$. The required values of $\mathrm{k}_{\perp}$ and $\mathrm{k}_{\|}$correspond to a doping level of 0.0090 for a paramagnetic system; however, if the ellipsoids represent a fully polarized electron gas, the corresponding doping level is 0.0045 , very close to the nominal doping level. The reported ordered moment of $0.07 \mu_{B}$ per carrier might reflect large magnetic fluctuations, noncollinearity of spins, orbital currents, or phase separation in the magnetic phase.

The origin of the magnetism has had no explanation except as an outgrowth of an excitonic undoped phase, which phase in $\mathrm{SrB}_{6}$ seems not to be supported by the data. At $x=0.005$ and higher, the electronic structure of the paramagnetic systems seems to be very simple. Inter-pocket $e-e$ nesting between inequivalent $\mathrm{X}$ points 
is the only $\omega \rightarrow 0$ nesting ( $\mathrm{Q} \neq 0$ ), and is maximum between points on their equators. If the Fermi surfaces were spheres, $\omega=0$ nesting would be perfect for $\mathrm{Q}=(1,1,0) \pi / a$ wavevectors, tending to drive CDW or SDW instabilities. Due to the eccentricity (i.e. $m_{t} \neq m_{l}$ ), these scattering processes are smeared by $\delta \mathrm{Q} \sim 2 \times 10^{-2} \pi / a$ for $\omega=0$ or by $\delta \omega \sim 30 \mathrm{meV}$ at $\mathrm{Q}$ itself. Moreover, the system is already magnetic above the degeneracy temperature, where nesting is an irrelevant process. At high $\mathrm{T}$ a nonzero - but rather small - density of holes will be thermally excited, leading perhaps to a charge-unbalanced, quasiclassical $e-h$ plasma, but the high temperature should also preclude formation of an exciton-fermion plasma.

To summarize, we have looked closely at the electronic structure of undoped and doped divalent hexaborides, and compared them with the available transport, thermal, optical, dHvA, and tunneling data. The behavior of $\mathrm{SrB}_{6}$ above $5 \mathrm{~K}$ is consistent with expectations, and energy scales, obtained from the band structure. The observed Fermi surface of $x=0.005$ doped $\mathrm{CaB}_{6}$ is most easily accounted for as the majority Fermi surface of a fully polarized electron gas, but this interpretation is not readily reconciled with the measured tiny moment.

We acknowledge helpful communications with Z. Fisk, R. G. Goodrich, and J. W. Allen during the course of this work, and comments on the manuscript by R. Monnier. This work was supported by an NSF-CONICET International Collaboration. W. E. P. was supported by National Science Foundation (NSF) Grant No. DMR9802076. Ruben Weht acknowledges support from Fundación Antorchas Grants No. A-13622/1-103 and A13661/1-27. Much of this work was done when the authors were at the Institute of Theoretical Physics in Santa Barbara, which is supported by NSF Grant No. PHY 9407194.

[1] D. P. Young et al., Nature 397, 412 (1999).

[2] M. E. Zhitomirsky, T. M. Rice, and V. I. Anisimov, Nature 402, 251 (1999); cond-mat/9904330.

[3] V. Barzykin and L. P. Gor'kov, Phys. Rev. Lett. 84, 2207 (2000).

[4] L. Balents and C. M. Varma, Phys. Rev. Lett. 84, 1264 (2000).

[5] L. V. Keldysh and Yu. V. Kopaev, Sov. Phys. Solid State 6, 2219 (1965).

[6] J. des Cloizeaux, J. Phys. Chem. Solids 26, 259 (1965).

[7] M. E. Zhitomirsky and T. M. Rice, cond-mat/9910272.

[8] H. R. Ott et al., Z. Phys. B 102, 337 (1997).

[9] D. Hall et al., Bull. Am. Phys. Soc. 44, 215 (1999); R. G. Goodrich, unpublished.

[10] A. Hasegawa and A. Yanase, J. Phys. C 12, 5431 (1979).

[11] S. Massidda et al., Z. Phys. B 102, 83 (1997).
[12] H. Ripplinger, K. Schwarz, and P. Blaha, J. Solid State Chem. 133, 51 (1997).

[13] P. Blaha, K. Schwarz, and J. Luitz, WIEN97, Vienna University of Technology, 1997. Improved and updated version of the original copyrighted WIEN code, which was published by P. Blaha, K. Schwarz, P. Sorantin, and S. B. Trickey, Comput. Phys. Commun. 59, 399 (1990).

[14] D. M. Ceperley and B. J. Alder, Phys. Rev. Lett. 45, 566 (1980), as parametrized by J. P. Perdew and Y. Wang, Phys. Rev. B 45, 13244 (1992).

[15] J. P. Perdew et al., Phys. Rev. B 46, 6671 (1992); J. P. Perdew, K. Burke, and M. Ernzerhof, Phys. Rev. Lett. 77, 3865 (1996).

[16] Z. Fisk, unpublished.

[17] B. Amsler et al., Phys. Rev. B 57, 8747 (1998).

TABLE I. Calculated Fermi surface properties of $\mathrm{SrB}_{6}$. F denotes the dHvA frequency and $m^{*}$ denotes the band masses. See text for notation.

\begin{tabular}{lccccc}
\hline \hline & $\mathrm{O}_{\text {lens }, 0}$ & $\mathrm{O}_{\text {lens }, 1}$ & $\mathrm{O}_{\text {ring, }, 0}$ & $\mathrm{O}_{\text {ring,out }}$ & $\mathrm{O}_{\text {ring,in }}$ \\
\hline $\mathrm{F}($ Tesla $)$ & 33 & 46 & 10 & 98 & 61 \\
$m_{b}^{*} / m_{o}$ & -0.35 & $\sim 0.2$ & 0.35 & 0.2 & -0.2 \\
\hline \hline
\end{tabular}

